KFKI-1988-05/A

HU ISSN 03685330

February 1988

\title{
CONTINUOUS QUANTUM MEASUREMENT AND ITÔ FORMALISM
}

\author{
L. Diósi \\ Central Research Institute for Physics \\ H-1525 Budapest 114, P.O.B. 49, Hungary
}

\begin{abstract}
A new quantum-stochastic differential calculus is derived for representing continuous quantum measurement of the position operator. Closed nonlinear quantum-stochastic differential equation is given for the quantum state of the observed particle. Possible physical realization of continuous position measurement is constructed.
\end{abstract}




\section{Introduction}

In 1982 Barchielli, Lanz and Prosperi suggested a theory of continuous quantum measurement ${ }^{1}$ including observables of continuous spectra such like, e.g., coordinate operator. Various equivalent formalisms were elaborated: path integrals ${ }^{1}$, characteristic functionals ${ }^{2}$, quantum-stochastic differential equations (QSDE's) ${ }^{3,4}$. Very recently, Ghirardi, Rimini and Weber have proposed the appealing unification of microscopic and macroscopic dynamics ${ }^{5}$ in the frame of a similar theory to that of continuous quantum measurements.

In this paper we suggest a simple quantum-stochastic formulation, à la Itô, for continuous quantum measurements ${ }^{1}$. It shows very close relationship to the Stratonowitch-calculus used by Gisin for discrete observables ${ }^{6}$. In Sect. 2 we outline the principles of continuous observation and present the proper nonlinear Itô-equations (which differ from Itô-equations of Ref. 4). The derivation of our equations is postponed to Sect. 3; in Sect. 4 we propose a possible physical model to which our formalism can presumably be applied. 


\section{Continuous position measurement, Itô-equations}

In order to reduce the amount of technical work we single out the simplest example and we consider the continuous measurement of the position $\hat{q}$ of a free particle of mass $m$ moving in one dimension.

Let the state vector $\psi$ satisfy the free Schrödinger equation; then the density operator $\hat{\rho} \equiv \psi \psi^{\dagger}$ obeys the following equation:

$$
\hat{\rho}=-(i / 2 m)\left[\hat{p}^{2}, \hat{\rho}\right] \quad ;
$$

$\hat{p}$ is the momentum operator canonically conjugated to the coordinate $\hat{q}$. Now, Refs. 1, 5 introduce instantaneous stochastic changes, too:

$$
\begin{aligned}
& \hat{\rho} \rightarrow[P(\bar{q})]^{-1} T_{\bar{q}}[\hat{\rho}] \equiv \\
& \equiv[P(\bar{q})]^{-1} \sqrt{\alpha / \pi} \exp \left[-\frac{1}{2} \alpha(\hat{q}-\bar{q})^{2}\right] \hat{\rho} \exp \left[-\frac{1}{2} \alpha(\hat{q}-\bar{q})^{2}\right]
\end{aligned}
$$

called measurement (or localization) processes which repeatedly occur at equidistant moments ${ }^{1}$ separated by $\Delta t$. In the above equation $\alpha$ denotes the accuracy parameter of measurement. The measured coordinate $\bar{q}$ is selected at random with the probability distribution

$$
P(\bar{q})=\operatorname{tr} T_{\bar{q}}[\hat{\rho}]=\sqrt{\alpha / \pi} \operatorname{tr} \hat{\rho} \exp \left[-\alpha(\hat{q}-\bar{q})^{2}\right]
$$


The Eqs. (2.1-3) prescribe a certain joint stochastic process for $\hat{\rho} \equiv \psi \psi^{\dagger}$ and $\bar{q}$. Barchielli et al. define the continuous measurement of the position $\hat{q}$ by taking the following limit

$$
\Delta t, \alpha \rightarrow 0, \alpha / \Delta t=\gamma=\text { const. }
$$

of the stochastic process. [Here we have to note that Ghirardi et al. ${ }^{5}$ suggest finite values for $\alpha$ and $\Delta t$, without requiring the limes (2.4). Nevertheless, they also notice that most physical characteristics of their theory depend only on the ration $\gamma=\alpha / \Delta t$.]

Observe that, while $\hat{\rho}$ is a function of the time $t$, the other stochastic variable $\bar{q}$ only makes sense for the instants of the measurement process (2.2). Let us extend the definition of $\bar{q}$ for all time: one can, e.g., identify $\bar{q}_{t}$ by the proper continuous zig-zag function of time. We introduce then a new variable $\bar{Q}$ instead of $\bar{q}$ :

$$
\bar{Q}_{t}=\int_{0}^{t} \bar{q}_{t^{\prime}} d t^{\prime} .
$$

In the next Section we shall prove that $\hat{\rho}$ and $\bar{Q}$ follow a Gaussian process. They obey the couple of stochastic differential equations; one is for the $\hat{\rho}$ valued quantum-stochastic process: 


$$
d \hat{\rho}=\left(-i\left[\hat{p}^{2} / 2 m, \hat{\rho}\right]-\frac{1}{4} \gamma[\hat{q},[\hat{q}, \hat{\rho}]]\right) d t+\{\hat{q}-\bar{q}, \hat{\rho}\} d \xi
$$

the other is for the measured coordinate:

$$
d \bar{Q}=\langle\hat{q}\rangle d t+\gamma^{-1} d \xi
$$

Here $\xi$ is a Wiener-process. The Itô-differential ${ }^{7} d \xi$ satisfies the following algebra:

$$
\begin{gathered}
\langle d \xi\rangle_{\mathrm{st}}=0 \\
d \xi d \xi=\frac{1}{2} \gamma d t \\
(d \xi)^{n}=0 \text { if } n=3,4, \ldots
\end{gathered}
$$

Through our paper $\langle$.$\rangle stands for the quantum expectation values while \langle.\rangle_{\text {st }}$ denotes stochastic means.

It is important to see that the QSDE (2.6) preserves the pure state property $\hat{\rho} \equiv \psi \psi^{\dagger}=\hat{\rho}^{2}$. In fact, it is enough to prove that $d \hat{\rho}^{2}=d \hat{\rho}$ provided $\hat{\rho}^{2}=\hat{\rho}$ at a given moment. We therefore substitute the QSDE (2.6) into the RHS of the identity $d \hat{\rho}^{2}=\{d \hat{\rho}, \hat{\rho}\}+d \hat{\rho} d \hat{\rho}$. Using the Itô-algebra (2.8) and the assumption $\hat{\rho}^{2}=\hat{\rho}$, we arrive at the identity $d \hat{\rho}^{2}=d \hat{\rho}$.

One may notice that the so-called measured coordinate $\bar{q}$ is not a good representative of the particle trajectory. From the Eqs. (2.5) and (2.7) one can formally get 


$$
\bar{q}=\langle\hat{q}\rangle+\gamma^{-1} \dot{\xi}
$$

which means that $\bar{q}$ is charged by a stationary white-noise making the graph $\bar{q}_{t}$ an awkward fractal $^{8}$ instead of a trajectory. This problem has already been emphasized in Refs. 1, 5 as well.

It is thus better to represent the trajectory by $\langle\hat{q}\rangle$ instead of $\bar{q}$. Consequently, our proposal is to concentrate on the nonlinear quantum-stochastic differential equation (QSDE) (2.6) [together with the Itô-algebra (2.8)]. This QSDE, in itself, will account for all the physical behaviour of the particle affected by the continuous coordinate measurement.

\section{Verification of Itô-equations}

In the present Section we are going to derive the stochastic differential equations $(2.6),(2.7)$ starting from Eqs. (2.1-4) of continuous position measurement ${ }^{1}$.

First we need the notion of stochastic mean for the measurement process $(2.2)$ :

$$
\langle\cdot\rangle_{\mathrm{st}} \equiv \int \cdot P(\bar{q}) d \bar{q}
$$

We introduce the change of our stochastic variables $\hat{\rho}$ and $\bar{Q}$ taken for one cycle of duration $\Delta t$ : 


$$
\begin{aligned}
& \Delta \hat{\rho}_{t} \equiv \hat{\rho}_{t+\Delta t}-\hat{\rho}_{t}= \\
& =-i\left[\hat{p}^{2} / 2 m, \hat{\rho}_{t}\right] \Delta t+\left[P_{t}\left(\bar{q}_{t}\right)\right]^{-1} T_{\bar{q}_{t}}\left[\hat{\rho}_{t}\right]-\hat{\rho}_{t}+\mathcal{O}\left[(\Delta t)^{2}\right] \\
& \Delta \bar{Q}_{t} \equiv \bar{Q}_{t+\Delta t}-\bar{Q}_{t}=\bar{q}_{t} \Delta t+\mathcal{O}\left[(\Delta t)^{2}\right]
\end{aligned}
$$

where we used Eqs. (2.1-2) and Eq. (2.5), respectively.

The continuous measurement process (2.1-4) is a Gaussian process with Itô-equations (2.6-8) if, in the limes (2.4), the moments of $\Delta \hat{\rho}$ and $\Delta \bar{Q}$ [c.f. Eqs. (3.2),(3.3)] satisfy the same algebra as the corresponding Itôdifferentials $d \hat{\rho}, d \bar{Q}$ [c.f. Eqs. (2.6),(2.7)]. Consequently, we have to prove the following asymptotics:

$$
\begin{aligned}
& (1 / \Delta t)\langle\Delta \hat{\rho}\rangle_{\mathrm{st}} \rightarrow(1 / d t)\langle d \hat{\rho}\rangle_{\mathrm{st}}=-i\left[\hat{p}^{2} / 2 m, \hat{\rho}\right]-\frac{1}{4} \gamma[\hat{q},[\hat{q}, \hat{\rho}]] \\
& (1 / \Delta t)\langle\Delta \bar{Q}\rangle_{\mathrm{st}} \rightarrow(1 / d t) d \bar{Q}=\langle\hat{q}\rangle \\
& (1 / \Delta t)\langle\Delta \hat{\rho} \otimes \Delta \hat{\rho}\rangle_{\mathrm{st}} \rightarrow(1 / d t) d \hat{\rho} \otimes d \hat{\rho}=\frac{1}{2} \gamma\{\hat{q}-\bar{q}, \hat{\rho}\} \otimes\{\hat{q}-\bar{q}, \hat{\rho}\} \\
& (1 / \Delta t)\langle\Delta \bar{Q} \Delta \bar{Q}\rangle_{\mathrm{st}} \rightarrow(1 / d t) d \bar{Q} d \bar{Q}=1 / 2 \gamma \\
& (1 / \Delta t)\langle\Delta \bar{Q} \Delta \hat{\rho}\rangle_{\mathrm{st}} \rightarrow(1 / d t) d \bar{Q} d \hat{\rho}=\frac{1}{2}\{\hat{q}-\bar{q}, \hat{\rho}\} \\
& (1 / \Delta t)\langle\text { higher than 2nd powers of } d \hat{\rho} \text { and /or } d \bar{Q}\rangle_{\mathrm{st}} \rightarrow 0 .
\end{aligned}
$$

The stochastic means on the LHS's must be taken at time $t$ (i.e. at the beginning of the interval $\Delta t$ ). The expressions on the very right have been calculated by using the stochastic differential equations (2.6),(2.7) and the Itô-algebra (2.8). To prove the above asymptotical relations, we are going to evaluate the LHS terms in turn. 
As for the first one, Eq. (3.4a) is known from previous works. Its proof is easy because the probability distribution $P(\bar{q})$ cancels from $\langle\Delta \hat{\rho}\rangle_{\text {st }}$. Thus, we refer the reader to the literature ${ }^{1,5}$ where the following master equation has been proved:

$$
d / d t\langle\Delta \hat{\rho}\rangle_{\mathrm{st}}=-i\left[\hat{p}^{2} / 2 m,\langle\Delta \hat{\rho}\rangle_{\mathrm{st}}\right]-\frac{1}{4} \gamma\left[\hat{q},\left[\hat{q},\langle\Delta \hat{\rho}\rangle_{\mathrm{st}}\right]\right] .
$$

Its special case, i.e. when $\langle\Delta \hat{\rho}\rangle_{\text {st }}=\psi \psi^{\dagger}=\hat{\rho}$ at time $t$, yields just the Eq. (3.4a).

To prove Eqs. (3.4b-f) we need the form of the distribution $P(\bar{q})$. In what follows we exploit translational invariance and choose a special coordinate system where $\langle\hat{q}\rangle=0$ for the give state $\hat{\rho}$ at time $t$; this choice makes our calculations simpler without loss of their generality. Then Eq. (2.3) yields the following asymptotical expansion:

$$
P(\bar{q})=\sqrt{\alpha / \pi} \exp \left(-\alpha \bar{q}^{2}\right)\left[1+\mathcal{O}(\alpha)+\mathcal{O}\left(\alpha^{2} \bar{q}^{2}\right)\right] .
$$

In this approximation, Eq. (3.1) yields

$$
\begin{aligned}
\langle\bar{q}\rangle_{\mathrm{st}} & =\mathcal{O}(\alpha) \\
\left\langle\bar{q}^{2}\right\rangle_{\mathrm{st}} & =(1 / 2 \alpha)+\mathcal{O}(1) .
\end{aligned}
$$

and, by virtue of Eq. (3.2), we obtain

$$
\Delta \hat{\rho}=\alpha \bar{q}\{\hat{q}, \hat{\rho}\}+\mathcal{O}\left(\alpha^{2} \bar{q}^{2}\right)
$$


We remind the reader that we have supposed $\langle\hat{q}\rangle=0$.

Let us evaluate each LHS term of Eqs. (3.4b-e) in turn, with the help of Eqs. (3.3), (3.7ab) and (3.8):

$(1 / \Delta t)\langle\Delta \bar{Q}\rangle_{\mathrm{st}} \rightarrow(1 / \Delta t)\langle\bar{q}\rangle_{\mathrm{st}} \Delta t \rightarrow 0$

$(1 / \Delta t)\langle\Delta \hat{\rho} \otimes \Delta \hat{\rho}\rangle_{\mathrm{st}} \rightarrow(1 / \Delta t) \alpha^{2}\left\langle\bar{q}^{2}\right\rangle_{\mathrm{st}}\{\hat{q}, \hat{\rho}\} \otimes\{\hat{q}, \hat{\rho}\} \rightarrow \frac{1}{2} \gamma\{\hat{q}, \hat{\rho}\} \otimes\{\hat{q}, \hat{\rho}\}$

$(1 / \Delta t)\langle\Delta \bar{Q} \Delta \bar{Q}\rangle_{\mathrm{st}} \rightarrow(1 / \Delta t)\left\langle\bar{q}^{2}\right\rangle_{\mathrm{st}}(\Delta t)^{2} \rightarrow 1 / 2 \gamma$

$(1 / \Delta t)\langle\Delta \bar{Q} \Delta \hat{\rho}\rangle_{\mathrm{st}} \rightarrow(1 / \Delta t) \alpha \Delta t\left\langle\bar{q}^{2}\right\rangle_{\mathrm{st}}\{\hat{q}, \hat{\rho}\} \rightarrow \frac{1}{2}\{\hat{q}, \hat{\rho}\}$

Recalling that $\langle\hat{q}\rangle=0$, the Eqs. (3.9) have provided the proof of Eqs. (3.4be). We shall not give a systematic proof to Eq. (3.4f). The reader may convince himself that the increasing powers of $\Delta t$ (or, equivalently, of $\alpha$ ) will not further be compensated by the higher moments of $\bar{q}$, and expressions like $1 / \Delta t\left\langle(\Delta \bar{Q})^{3}\right\rangle_{\mathrm{st}}, 1 / \Delta t\left\langle(\Delta \hat{\rho} \otimes \Delta \hat{\rho} \otimes \Delta \hat{\rho}\rangle_{\mathrm{st}}\right.$, e.t.c. will tend to the zero. Besides, this is the genuine feature if Gaussian processes.

So we have presented the proof of Itô-equations $(2.6),(2.7)$ for the continuous measurement (2.1-4) of a free particle. 


\section{Possible physical model}

We try to construct a physical model whose state would follow the quantum stochastic process described by the QSDE (2.6). Let us suppose that our test particle is not free but immersed in a parallel stream of light particles (e. g. of photons). We consider the quantum motion of the probe only in the transversal plane. This model has been stimulated by a similar discussion given recently by Joos and Zeh ${ }^{9}$. (Meanwhile, a more formal treatment has been shown by Caves and Milburn ${ }^{10}$ ).

At the beginning, let us start the probe with a given pure quantum state $\hat{\rho}=\psi \psi^{\dagger}$. It obeys to the free Schrödinger-equation (2.1) until the first collision with a photon occurs. The quantum state of the probe bears an instantaneous random change. It is very crucial to realize that this change (collapse) may be identified if we detect the scattered photon. The set of

possible collapses depends on the photon measuring apparatus. If, e.g., we observe the scattered photon through an optical lens, we can detect the current position of the probe. The non-unitary localization process (2.2) may, at least qualitatively, represent the collapse of the quantum state where $1 / \sqrt{\alpha}$ is the resolution of the optical device in the transverse plane. The operator $\hat{q}$ stands for the transverse coordinate: the probability distribution of the measured transverse position $\bar{q}$ of the probe is given by (2.3). 
The optimal resolution $1 / \sqrt{\alpha}$ and the repetition frequency $1 / \Delta t$ of single measurements depend on the wave number $k$ of photons, on the intensity $I$ of their stream and on the total cross section $\sigma$ of photon scattering on the probe:

$$
1 / \sqrt{\alpha} \approx 1 / k, \quad 1 / \Delta t \approx \sigma I
$$

Now, provided the current wave function of the probe is such that 1) its transverse width is much smaller than the resolution $1 / \sqrt{\alpha}, 2$ ) its relative change during $\Delta t$ in between collisions is small and 3) we consider the probe properties on time scales larger than $\Delta t$ then, we can exploit the property of the limes (2.4). Hence, one may expect that the QSDE (2.6) will account for the change of the probe's quantum state $\hat{\rho}$ and, furthermore, the observed position $\bar{q}$ is governed by the stochastic differential equation (2.7).

It would be interesting to see experimentally the fractal nature of the observed "trajectory". We should remember, that this fractality as well as the validity of the Eqs. (2.6-7) break down at time scales equal or less than $\Delta t$.

Finally, we briefly discuss the alternative continuous measurement on the very same system. Let us remove the lens from our measuring apparatus and observe thus the momentum of the scattered photon. The the quantum state acquires the unitary change 


$$
\hat{\rho} \rightarrow \exp (i \Delta \bar{p} \hat{q}) \hat{\rho} \exp (-i \Delta \bar{p} \hat{q})
$$

instead of the collapse (2.2): here $\Delta \bar{p}$ is the measured (transverse) momentum transfer whose probability distribution depends on the differential cross section of the collision. We note without proof that, in the limes (2.4), the following QSDE fulfils for the quantum state of the probe:

$$
d \hat{\rho}=\left(-i\left[\hat{p}^{2} / 2 m, \hat{\rho}\right]-\frac{1}{4} \gamma[\hat{q},[\hat{q}, \hat{\rho}]]\right) d t-i[\hat{q}, \hat{\rho}] d \xi .
$$

This QSDE is linear and it corresponds to unitary evolution of the probe state in the effective white-noise potential $\hat{q} \dot{\xi}$ of the photon "heat bath", c.f. Refs. 11, 12 .

Note that both QSDE's (2.6) and (4.3) yield the same master equation (3.5) since the observation of scattered photon does not make any change to the statistical operator $\langle\hat{\rho}\rangle_{\text {st }}$ of the probe. The general pure state QSDE (i.e. preserving $\hat{\rho} \equiv \hat{\rho}^{2}$ ) for the same system has the following stochastic term:

$$
\cos \beta\{\hat{q}-\langle\hat{q}\rangle, \hat{\rho}\} d \xi-i \sin \beta[\hat{q}, \hat{\rho}] d \xi
$$

with arbitrary real $\beta$. In particular, this would correspond to the simultaneous unsharp measurement of the position and the momentum transfer of the 
probe. (We mention the non-Gaussian pure state stochastic process ${ }^{13}$ which could probably be connected with some even more sophisticated measurement of the scattered photon.)

\section{Concluding remarks}

The formal extension of the Itô-equations (2.6-8) for the continuous measurement of several (not necessarily commuting) operators seems to be straightforward. It would, nevertheless, be desirable to recapitulate the proof starting, e.g., from the mathematical representation of continuous measurements presented in Refs. 3, 4.

In a forthcoming paper we shall analyze the evolution of the QSDE (2.6) and we shall show that it possess a unique stationarily localized solution suitable to represent classical trajectories.

The author thanks Profs. A. Frenkel and B. Lukács for helpful discussions and Prof. P. Hraskó for useful remarks. 


\section{References}

[1] A.Barchielli, L.Lanz and G.M.Prosperi, Nuovo Cim. 72B, 79 (1982)

[2] A.Barchielli, Nuovo Cim. 74B, 113 (1983)

[3] A.Barchielli and G.Lupieri, J.Math.Phys. 26, 2222 (1985)

[4] A.Barchielli, Phys.Rev. D34, 1642 (1986)

[5] G.C.Ghirardi, A.Rimini and T.Weber, Phys.Rev. D34, 470 (1986)

[6] N.Gisin, Phys.Rev.Lett. 82, 1657 (1984)

[7] L.Arnold, Stochastic Differential Equations: Theory and Applications (Wiley, New York, 1974)

[8] L.F.Abbot and M.B.Wise, Am.J.Phys. 49, 37 (1981)

[9] E.Joos and H.D.Zeh, Z.Phys. B59, 223 (1985)

[10] C.M.Caves and G.J.Milburn, Phys.Rev. A36, 5543 (1987)

[11] V. Gorini et al., Rep. Math. Phys. 13149 (1987)

[12] V.R.Chechetkin and V.S.Lutovinov, J.Phys. A20, 4757 (1987)

[12] L.Diósi, Phys.Lett. 112A, 288 (1985) 